\title{
Exact quark-mass dependence of the Higgs-gluon form factor at three loops in QCD
}

\section{Michat Czakon and Marco Niggetiedt}

Institut für Theoretische Teilchenphysik und Kosmologie, RWTH Aachen University, Aachen D-52056, Germany

E-mail: mczakon@physik.rwth-aachen.de, marco.niggetiedt@rwth-aachen.de

ABSTRACT: We determine the three-loop form factor parameterising the amplitude for the production of an off-shell Higgs boson in gluon fusion in QCD with a single massive quark. The result is obtained via a numerical solution of a system of differential equation for the occurring master integrals. The solution is also used to determine the high-energy and threshold expansions of the form factor. Our findings may be used for the evaluation of virtual corrections generated by top-quark and b-quark loops in Higgs boson hadroproduction cross sections at next-to-next-to-leading order.

KeYwords: Higgs Physics, Perturbative QCD

ArXiv EPrint: 2001.03008 


\section{Contents}

1 Introduction 1

2 Finite remainders 3

3 Technicalities $\quad 6$

4 Results 18

5 Conclusions and outlook $\quad 10$

$\begin{array}{ll}\text { A Large-mass expansion } & 14\end{array}$

$\begin{array}{lr}\text { B Threshold expansion } & 18\end{array}$

$\begin{array}{ll}\text { C High-energy expansion } & 20\end{array}$

$\begin{array}{ll}\text { D Supplemental material } & 21\end{array}$

\section{Introduction}

Recent interest in the Higgs-gluon form factor is stimulated primarily by studies on the precision of cross section predictions for various hadron-collider processes involving an intermediate Higgs boson [1]. Indeed, the amplitude $g g \rightarrow H$ contributes to both singleand double-Higgs production with subsequent Higgs decay to a pair of fermions or offshell gauge bosons. In consequence, applications require the knowledge of the form factor for arbitrary virtualities, and the uncertainty induced by the standard use of the infinite top-quark mass limit plays a non-negligible role.

In pure $\mathrm{QCD}$, the evaluation of the form factor is complicated by the fact that the process is loop induced. Nevertheless, exact two-loop results for arbitrary quark masses have been available since refs. [2-5]. Improvement over the current accuracy of cross section predictions requires the knowledge of the form factor at three-loop order. This is quite a challenging problem that has been first attacked with the help of the large-mass expansion in the top-quark mass $[6,7]$. A large-mass expansion has even been derived at four-loop order [8]. Further progress at three-loops has been recently achieved using Padé approximants [9] exploiting partial knowledge of the form factor's behaviour around threshold [10]. While a complete result for the form factor at this order remains elusive, an exact result in terms of harmonic polylogarithms has been obtained for contributions involving a massless-quark loop [11]. The diagrams contributing to the latter calculation are depicted in figure 1. The same diagrams also contribute with two massive quark loops. 

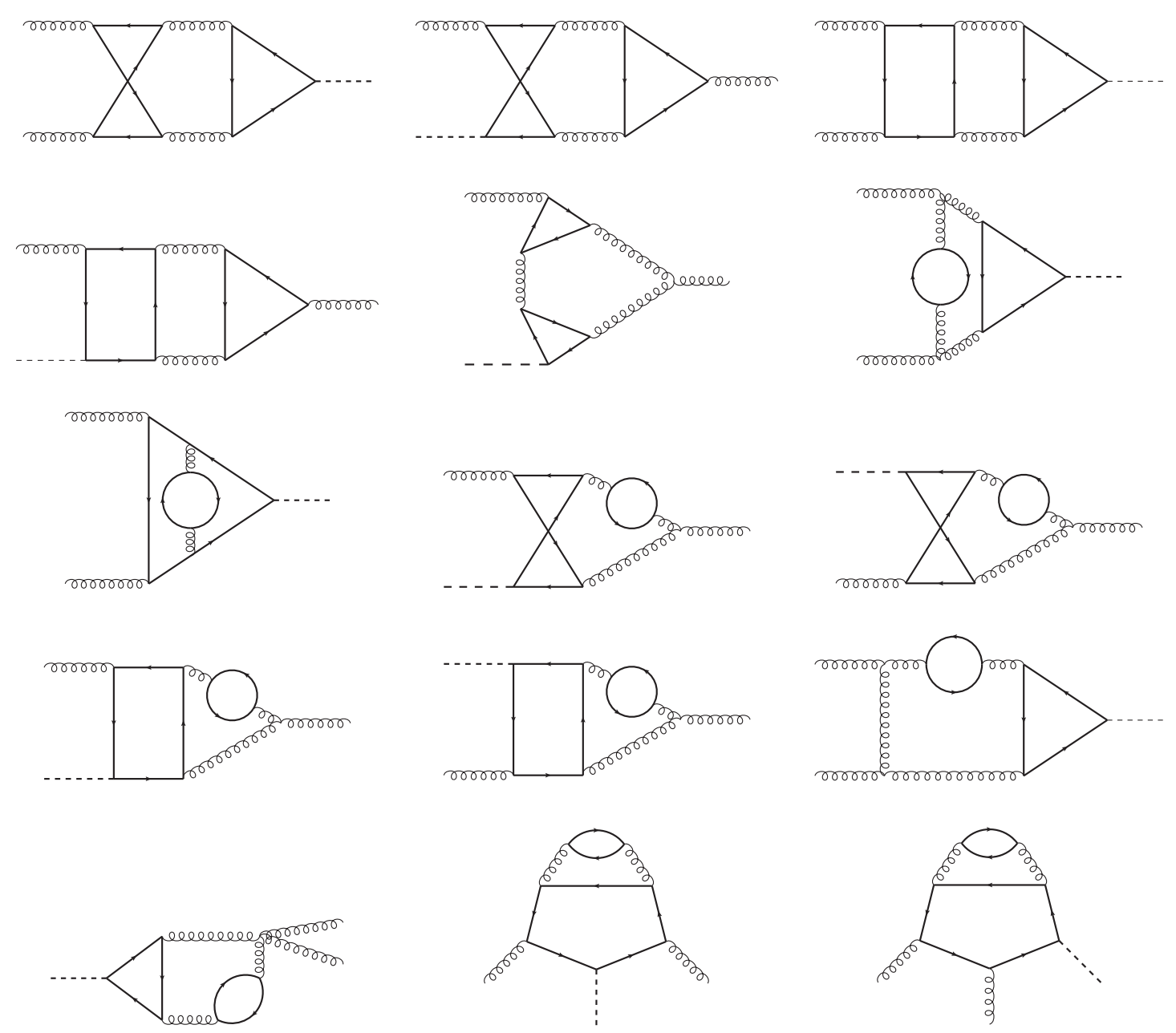

Figure 1. Complete set of Feynman diagrams with two fermion loops contributing to the Higgsgluon form factor at three-loop order. The fermion loop connected to the Higgs-boson line corresponds to a massive quark. The quark of the second fermion loop may be either massive or massless.

In the present publication, we present an exact result for the form factor in QCD with a single massive quark. In particular, we compute the diagrams of figure 1 with both quark loops with the same flavour, as well as the complete set of diagrams with only one massivequark loop. A result in QCD with several massive quarks would still require a calculation of the diagrams figure 1 with massive quarks of different flavour.

Our results are certainly necessary to answer the question whether Padé approximants are indeed sufficient phenomenologically as claimed in ref. [9]. Independently, the knowledge of exact quark mass dependence of the form factor opens the possibility of including b-quark mass effects exactly.

The paper is organised as follows. In the next section, we introduce our conventions and define finite remainders of the form factor after infrared renormalisation. We use this opportunity to provide explicit formulae for the scale dependence of the form factor as well. We subsequently describe the methodology that has allowed us to obtain not only a 
high precision numerical result but also high-order expansions around the three physical singularities: infinite quark mass (large-mass expansion), intermediate-quark production threshold (threshold expansion) and vanishing quark mass (high-energy expansion). Finally, we present our results and compare them to previous work, in particular, the Padé approximants of ref. [9]. This main text is closed with conclusions and outlook. The three expansions are reproduced in separate appendices. The last appendix presents the contents of the supplementary material that contains our results in electronic form.

\section{Finite remainders}

Consider the amplitude for the fusion of two gluons of momenta $p_{1,2}$, helicities $\lambda_{1,2}$ and adjoint-representation colors $a_{1,2}$, followed by the production of one, possibly off-shell, Higgs boson:

$$
\begin{aligned}
-i \mathcal{M}\left[g\left(p_{1}, \lambda_{1}, a_{1}\right)+g\left(p_{2}, \lambda_{2}, a_{2}\right) \rightarrow H\right] & \equiv \\
& i \delta^{a_{1} a_{2}}\left[\left(\epsilon_{1} \cdot p_{2}\right)\left(\epsilon_{2} \cdot p_{1}\right)-\left(\epsilon_{1} \cdot \epsilon_{2}\right)\left(p_{2} \cdot p_{1}\right)\right] \frac{1}{v} \frac{\alpha_{s}}{\pi} \mathcal{C} .
\end{aligned}
$$

Here, $v$ is the Higgs-doublet Vacuum Expectation Value. The coupling of a single quark field, $Q$, of mass $M \neq 0$ to the Higgs-boson field, $H$, is given by the tree-level Lagrangian term $-M \bar{Q} Q H / v$. Finally, the gluon polarisation vectors are normalised as follows:

$$
\epsilon_{i} \equiv \epsilon\left(\boldsymbol{p}_{i}, \lambda_{i}\right), \quad \epsilon_{i} \cdot p_{i}=0, \quad \epsilon_{i} \cdot \epsilon_{i}^{*}=-1, \quad i=1,2 .
$$

The Form Factor $\mathcal{C}$ is expanded in the strong coupling constant, $\alpha_{s}$, and the number of massless quark flavors, $n_{l}$ :

$$
\mathcal{C}=\mathcal{C}^{(0)}+\frac{\alpha_{s}}{\pi} \mathcal{C}^{(1)}+\left(\frac{\alpha_{s}}{\pi}\right)^{2} \mathcal{C}^{(2)}+\mathcal{O}\left(\alpha_{s}^{3}\right), \quad \mathcal{C}^{(n)}=\sum_{k=0}^{n} \mathcal{C}^{(n, k)} n_{l}^{k}
$$

The strong coupling is defined in the $\overline{\mathrm{MS}}$ scheme with massive-quark decoupling. Its dependence on the renormalisation scale $\mu$ is given by the $\beta$-function for $n_{l}$ massless quarks, $\alpha_{s} \equiv \alpha_{s}^{\left(n_{l}\right)}(\mu)$. Contributions $\mathcal{C}^{(n, n)} \neq 0, n>0$ are only due to coupling constant renormalisation. The massive-quark mass, $M$, is defined in the on-shell scheme implying the same for the Yukawa coupling. The dimensionless form-factor expansion coefficients depend on two variables only:

$$
\begin{aligned}
\mathcal{C}^{(n, k)} & \equiv \mathcal{C}^{(n, k)}\left(z, L_{\mu}\right), \\
z & \equiv \frac{s}{4 M^{2}}+i 0^{+}, \quad L_{\mu} \equiv \ln \left(-\frac{\mu^{2}}{s+i 0^{+}}\right), \quad s \equiv\left(p_{1}+p_{2}\right)^{2} .
\end{aligned}
$$

The leading contribution is:

$$
\mathcal{C}^{(0)}=\mathcal{C}^{(0,0)}=T_{F} \frac{1}{z}\left\{1-\left(1-\frac{1}{z}\right)\left[\frac{1}{2} \ln \left(\frac{\sqrt{1-1 / z}-1}{\sqrt{1-1 / z}+1}\right)\right]^{2}\right\}
$$


In the limit $M \rightarrow \infty$ :

$$
\mathcal{C}^{(0)}[z=0]=\frac{1}{3} .
$$

Hence, the amplitude eq. (2.1) may be obtained at $M \rightarrow \infty$ from the Higgs-EffectiveTheory tree-level Lagrangian:

$$
\mathcal{L}_{\mathrm{HET}}^{(0)}=\frac{\alpha_{s}}{12 \pi} \frac{H}{v} G_{\mu \nu}^{a} G^{a \mu \nu},
$$

where $G_{\mu \nu}^{a}$ is the standard QCD field-strength tensor, $\mathcal{L}_{\mathrm{QCD}}=-1 / 4 G_{\mu \nu}^{a} G^{a \mu \nu}+\mathcal{L}_{\text {matter }}$.

Beyond leading order, the form factor is infrared divergent after renormalisation. The results presented in this publication correspond to Conventional Dimensional Regularisation with space-time dimension $d=4-2 \epsilon$. The infrared divergences may be factorised yielding the Finite Remainder, $\mathcal{C}_{I}$, of the form factor:

$$
\mathcal{C}_{I} \equiv I \mathcal{C},
$$

where the two-loop I-operator of Catani [12] (see ref. [13] for the specific case of the Higgsgluon form factor) is given by:

$$
\begin{aligned}
I= & 1-\frac{\alpha_{s}}{2 \pi} I^{(1)}-\left(\frac{\alpha_{s}}{2 \pi}\right)^{2} I^{(2)}, \\
I^{(1)} \equiv & I^{(1)}(\epsilon)=-\left(-\frac{\mu^{2}}{s+i 0^{+}}\right)^{\epsilon} \frac{e^{\epsilon \gamma_{E}}}{\Gamma(1-\epsilon)}\left[\frac{C_{A}}{\epsilon^{2}}+\frac{b_{0}}{2 \epsilon}\right], \\
I^{(2)}= & -\frac{1}{2} I^{(1)}(\epsilon)\left(I^{(1)}(\epsilon)+\frac{b_{0}}{\epsilon}\right)+\frac{e^{-\epsilon \gamma_{E}} \Gamma(1-2 \epsilon)}{\Gamma(1-\epsilon)}\left(\frac{b_{0}}{2 \epsilon}+K\right) I^{(1)}(2 \epsilon) \\
& +\left(-\frac{\mu^{2}}{s+i 0^{+}}\right)^{2 \epsilon} \frac{e^{\epsilon \gamma_{E}}}{\Gamma(1-\epsilon)} \frac{H_{g}}{2 \epsilon},
\end{aligned}
$$

with the first two coefficients of the QCD $\beta$-function:

$$
b_{0}=\frac{11}{3} C_{A}-\frac{4}{3} T_{F} n_{l}, \quad b_{1}=\frac{34}{3} C_{A}^{2}-\frac{20}{3} C_{A} T_{F} n_{l}-4 C_{F} T_{F} n_{l},
$$

and:

$$
\begin{aligned}
K & =\left(\frac{67}{18}-\frac{\pi^{2}}{6}\right) C_{A}-\frac{10}{9} T_{F} n_{l} \\
H_{g} & =\left(\frac{5}{12}+\frac{11 \pi^{2}}{144}+\frac{\zeta_{3}}{2}\right) C_{A}^{2}+\left(-\left(\frac{58}{27}+\frac{\pi^{2}}{36}\right) C_{A}+C_{F}+\frac{20}{27} T_{F} n_{l}\right) T_{F} n_{l} .
\end{aligned}
$$

In general, $\mathcal{C}_{I}^{(n, n)} \neq 0, n>0$. However:

$$
\mathcal{C}_{I}^{(1,1)}\left[L_{\mu}=0\right]=0, \quad \mathcal{C}_{I}^{(2,2)}\left[L_{\mu}=0\right]=\frac{\pi^{2}}{864} \mathcal{C}^{(0)} .
$$

Just as the form factor itself, the $I$-operator, eq. (2.10), is independent of the scale $\mu$ (up to two-loop order of course). In consequence:

$$
\frac{\mathrm{d} \ln \mathcal{C}_{I}}{\mathrm{~d} \ln \mu}=\frac{\mathrm{d} \ln I}{\mathrm{~d} \ln \mu}+\frac{\mathrm{d} \ln \mathcal{C}}{\mathrm{d} \ln \mu}=0 .
$$


The dependence of the finite remainder on the scale logarithm, $L_{\mu}$, is thus given by the $\beta$-function only: ${ }^{1}$

$$
\begin{aligned}
& \mathcal{C}_{I}^{(1)}=\mathcal{C}_{I}^{(1)}\left[L_{\mu}=0\right]+\frac{b_{0}}{4} \mathcal{C}^{(0)} L_{\mu} \\
& \mathcal{C}_{I}^{(2)}=\mathcal{C}_{I}^{(2)}\left[L_{\mu}=0\right]+\frac{b_{0}}{2} \mathcal{C}_{I}^{(1)}\left[L_{\mu}=0\right] L_{\mu}+\frac{b_{1}+b_{0}^{2} L_{\mu}}{16} \mathcal{C}^{(0)} L_{\mu}
\end{aligned}
$$

A different finite remainder, $\mathcal{C}_{Z}$, is obtained if the factorisation of infrared divergences is performed in the $\overline{\mathrm{MS}}$ scheme [14]. Define:

$$
\mathcal{C}_{Z} \equiv Z^{-1} \mathcal{C}
$$

with:

$$
\frac{\mathrm{d} \ln Z^{-1}}{\mathrm{~d} \ln \mu} \equiv \Gamma \equiv-C_{A} \gamma_{\text {cusp }} L_{\mu}+2 \gamma_{g} .
$$

The solution at two-loops is:

$$
\begin{aligned}
\ln Z^{-1} & =-\frac{\alpha_{s}}{4 \pi}\left(\frac{\Gamma_{0}^{\prime}}{4 \epsilon^{2}}+\frac{\Gamma_{0}}{2 \epsilon}\right)-\left(\frac{\alpha_{s}}{4 \pi}\right)^{2}\left(-\frac{3 b_{0} \Gamma_{0}^{\prime}}{16 \epsilon^{3}}+\frac{\Gamma_{1}^{\prime}-4 b_{0} \Gamma_{0}}{16 \epsilon^{2}}+\frac{\Gamma_{1}}{4 \epsilon}\right), \\
\Gamma^{\prime} & \equiv \frac{\partial \Gamma}{\partial \ln \mu}=-2 C_{A} \gamma_{\text {cusp }}, \quad \Gamma \equiv \frac{\alpha_{s}}{4 \pi} \Gamma_{0}+\left(\frac{\alpha_{s}}{4 \pi}\right)^{2} \Gamma_{1},
\end{aligned}
$$

with the anomalous dimensions:

$$
\begin{aligned}
\gamma_{\text {cusp }} & =\frac{\alpha_{s}}{\pi}+\left(\frac{\alpha_{s}}{\pi}\right)^{2} \frac{K}{2} \\
\gamma_{g} & =-\frac{\alpha_{s}}{4 \pi} b_{0}+\left(\frac{\alpha_{s}}{4 \pi}\right)^{2}\left[\left(-\frac{692}{27}+\frac{11 \pi^{2}}{18}+2 \zeta_{3}\right) C_{A}^{2}+\left(\left(\frac{256}{27}-\frac{2 \pi^{2}}{9}\right) C_{A}+4 C_{F}\right) T_{F} n_{l}\right] .
\end{aligned}
$$

Since the dependence on the highest-power of $n_{l}$ in eq. (2.3) is only due to the pure poles in the minimal ultraviolet renormalisation constant $Z_{\alpha_{s}}$, it must be cancelled by the, equally minimal, constant $Z$. Thus:

$$
\mathcal{C}_{Z}^{(n, n)}=0 .
$$

The scale dependence of $\mathcal{C}_{Z}$, on the other hand, is non-trivial:

$$
\frac{\mathrm{d} \ln C_{Z}}{\mathrm{~d} \ln \mu}=\frac{\mathrm{d} \ln Z^{-1}}{\mathrm{~d} \ln \mu}+\frac{\mathrm{d} \ln \mathcal{C}}{\mathrm{d} \ln \mu}=\Gamma .
$$

The conversion between the two infrared schemes is achieved with the help of:

$$
\mathcal{C}_{Z}=(I Z)^{-1} \mathcal{C}_{I}
$$

\footnotetext{
${ }^{1}$ Notice that the $I$-operator of ref. [11] (see eq. (3.7b) of that publication) is missing a scale-dependent factor in the $H_{g}$-term (compare to eq. (4.38) of ref. [13]). With this difference, the $I$-operator of ref. [11] is not scale invariant and $\mathcal{C}_{I}^{(2)}$ contains an additional contribution to the single scale-logarithm term, $H_{g} / 4 \mathcal{C}^{(0)} L_{\mu}$.
} 
Explicitly:

$$
\begin{aligned}
&(I Z)^{-1}=1+\frac{\alpha_{s}}{\pi}\left\{\frac{\pi^{2}}{24} C_{A}+\left[-\frac{11}{12} C_{A}+\frac{1}{3} T_{F} n_{l}\right] L_{\mu}-\frac{1}{4} C_{A} L_{\mu}^{2}\right\} \\
&+\left(\frac{\alpha_{s}}{\pi}\right)^{2}\left\{-\left(\frac{\pi^{2}}{64}+\frac{11 \zeta_{3}}{96}\right) C_{A}^{2}+\left(\left(\frac{17 \pi^{2}}{864}+\frac{\zeta_{3}}{24}\right) C_{A}-\frac{\pi^{2}}{216} T_{F} n_{l}\right) T_{F} n_{l}\right. \\
&+ {\left[\left(-\frac{173}{108}+\frac{11 \pi^{2}}{288}+\frac{\zeta_{3}}{8}\right) C_{A}^{2}+\left(\left(\frac{16}{27}-\frac{\pi^{2}}{72}\right) C_{A}+\frac{1}{4} C_{F}\right) T_{F} n_{l}\right] L_{\mu} } \\
&+ {\left[\left(-\frac{67}{144}+\frac{\pi^{2}}{96}\right) C_{A}^{2}+\frac{5}{36} C_{A} T_{F} n_{l}\right] L_{\mu}^{2}+\left[\frac{11}{72} C_{A}^{2}-\frac{1}{18} C_{A} T_{F} n_{l}\right] L_{\mu}^{3} } \\
&+\left.\frac{1}{32} C_{A}^{2} L_{\mu}^{4}\right\} .
\end{aligned}
$$

For instance, this result allows to obtain eqs. (2.13) and the scale dependence of $\mathcal{C}_{Z}$ after using eqs. (2.15).

Finally, let us note that our results can be used to obtain the three-loop form factor before factorisation of the infrared divergences with the help of the two-loop result provided to $\mathcal{O}\left(\epsilon^{2}\right)$ in ref. [15].

\section{Technicalities}

The three-loop diagrams corresponding to the amplitude eq. (2.1) have been reduced to a set of (master) integrals, $M_{i}(z, \epsilon)$, via Integration-By-Parts identities [16] with the help of a $\mathrm{C}++$ implementation [17] of the Laporta algorithm [18]. The same reduction has also been exploited to construct a system of first-order homogeneous linear differential equations $[19,20]$ :

$$
\frac{\mathrm{d} M_{i}(z, \epsilon)}{\mathrm{d} z} \equiv \sum_{j} A_{i j}(z, \epsilon) M_{j}(z, \epsilon),
$$

where the coefficients $A_{i j}(z, \epsilon)$ are rational functions in $z$ and $\epsilon$. Truncated $\epsilon$-expansions have been subsequently substituted to represent the master integrals. A large-mass expansion (see below) of each $M_{i}$ has been used to determine the lowest power of $\epsilon, \underline{n}_{i}$, with non-vanishing coefficient, while the amplitude and the differential equations have been used to determine the highest power of $\epsilon, \bar{n}_{i}$, necessary to obtain the amplitude at $\mathcal{O}\left(\epsilon^{0}\right)$. Let the coefficients of the truncated $\epsilon$-expansions be denoted with $I_{k}(z)$ :

$$
M_{i}(z, \epsilon) \equiv \sum_{l=0}^{\bar{n}_{i}-\underline{n}_{i}} \epsilon^{\underline{\underline{n}}_{i}+l} I_{\underline{k}_{i}+l}(z),
$$

where $\underline{k}_{i}$ have been chosen to avoid overlap of the $k$-indices of the expansion coefficients $I_{k}$ of different master integrals. The coefficients $I_{k}$ satisfy a system of first-order homogeneous linear differential equations derived from eqs. (3.1):

$$
\frac{\mathrm{d} I_{k}(z)}{\mathrm{d} z} \equiv \sum_{l} B_{k l}(z) I_{l}(z),
$$




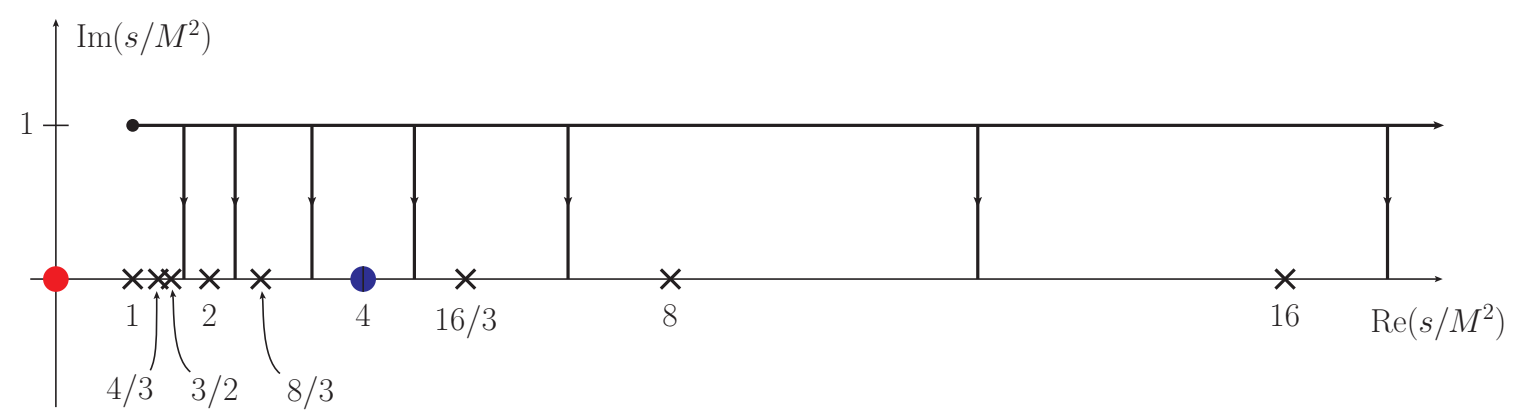

Figure 2. Contours for the numerical solution of the differential equations for the master integrals. The points on the abscissa correspond to singularities of the differential equations. Every time a contour reaches the real axis, the interval between singularities is explored in both directions.

where the coefficients $B_{k l}(z)$ are rational functions in $z$. Instead of seeking an analytic solution of eqs. (3.3), we have solved the system numerically as proposed originally in ref. [21] and first applied to a physical problem in ref. [22]. To this end, we have used the Boost [23] library odeint. In particular, we have chosen the Bulirsch-Stoer algorithm, bulirsch_stoer_dense_out. In order to keep the numerical precision of the results under control, we have used the Boost library multiprecision with a gmp/mpc backend. The floating point containers were requested to represent 100 decimal digits. A local error of $10^{-40}$ has been requested from the differential equation solution.

The numerical solution of eqs. (3.3) requires a boundary value for each $I_{k}$. In order to obtain these, we have used a high-order large-mass expansion, see e.g. [24], around $z=0$. The expansion must have unit radius of convergence ${ }^{2}$ in $z$, since the nearest singularity of the master integrals is at $z=1$. The expansion has been obtained using diagrammatic methods for the first few coefficients. It has been subsequently extended with the help of the differential equations. As boundary point, we have chosen $z=1 / 4(1+i)$, well within the radius of convergence. Because of the presence of singularities in the coefficients $B_{k l}$, we have used evolution contours shown in figure 2. An additional solution has also been obtained starting from $z=1 / 4(0.7+0.7 i)$ in order to control the error of the final result.

Having high-precision values of the master integrals allows to obtain expansions around arbitrary points, even around singularities. In the course of the present work, we have obtained threshold and high-energy expansions. They are necessary to evaluate the threeloop coefficient of the form factor in the vicinity of $z=1$ and $1 / z=0$ respectively. In general, expansions of $I_{k}$ are of power-log type, since an expansion in $\epsilon$ of the master integrals has already been performed:

$$
I_{k}(z(y)) \equiv \sum_{l=\underline{l}_{k}}^{\infty} \sum_{m=\underline{m}_{k}}^{\bar{m}_{k}} c_{k l m} y^{l} \ln ^{m} y,
$$

where $\underline{l}_{k}, \underline{m}_{k}, \bar{m}_{k} \in \mathbb{Z}$, and $y=\sqrt{1-z}$ for the threshold expansion, while $y=1 / z$ for the high-energy expansion. In practice, the expansions are truncated at an affordable order

\footnotetext{
${ }^{2}$ Strictly speaking, this is a power-log expansion with singularity at $z=0$. The convergence considerations apply to the coefficients of the logarithms, $\ln ^{m} z$, which are analytic in $z$.
} 
considering the available computing ressources. For each $I_{k}$, only one $c_{k} \equiv c_{k l m}$ for some $l$ and $m$, is necessary to make the solution of eqs. (3.3) unique. Since eqs. (3.3) are linear, there is:

$$
I_{k}(z(y)) \equiv \sum_{l} F_{k l}(y) c_{l} \quad \Longrightarrow \quad c_{k}=\sum_{l}\left(F^{-1}\right)_{k l}(y) I_{l}(z(y))
$$

In order to obtain $c_{k l m}$ and thus also $F_{k l}(y)$, we have used an efficient $\mathrm{C}++$ software that was originally developed for ref. [25]. Upon choosing a suitable $y$ point where the threshold or the high-energy expansion has excellent convergence, we were able to obtain $c_{k}$ with high precision.

\section{Results}

Since the scale logarithms of the three-loop coefficient of the finite remainder are entirely determined from the analytically known lower order results, see eqs. (2.15), we only present our findings at $L_{\mu}=0$.

We first note that our result for $\mathcal{C}_{I}^{(2,1)}$ agrees perfectly with ref. [11]. Remains to compare with the Padé approximants of ref. [9] for $\mathcal{C}^{(2)}$. A comparison for the case of five massless quarks is presented in figure 4. We observe that the uncertainty estimates of the approximants are reliable over most of the range of $z$. Slightly larger deviations are observed for the $n_{l}=0$ case as demonstrated in figure 5. An improvement of the Padé approximants has recently appeared in the proceedings [26]. The respective plots are also shown in figures 4 and 5 . Clearly, the agreement with the exact result is worse for $n_{l}=5$ and better for $n_{l}=0$.

In order to understand the phenomenological relevance of the difference between the exact result and its Padé approximation for $n_{l}=0$, we consider the quantity:

$$
\Delta^{(2,0)} \equiv\left|\left(\frac{\alpha_{s}}{\pi}\right)^{2} \frac{2 \operatorname{Re}\left[\left(\left.\mathcal{C}_{I}^{(2,0)}\right|_{[6,1]-\text { Padé }}-\mathcal{C}_{I}^{(2,0)}\right) \mathcal{C}^{(0)}\right]}{\left|\mathcal{C}^{(0)}\right|^{2}}\right|
$$

as a proxy for the error induced on the partonic cross section. We acknowledge the limitations of $\Delta^{(2,0)}$ in this respect due to the size of the real-radiation corrections to the cross section at higher orders. We expect that the actual effect is about $1 / 2$ of $\Delta^{(2,0)}$, at least for a top-quark loop. For simplicity, we fix the value of the strong coupling at $\alpha_{s}=0.1$. $\Delta^{(2,0)}$ is plotted in figure 3. Assuming an off-shell Higgs-boson with a partonic center-ofmass energy, $\sqrt{s}$, of up to $1 \mathrm{TeV}$ produced through a top-quark loop, there is $\Delta^{(2,0)}<1 \%$. Hence, the Padé approximant provides an excellent approximation for top-quark loops. On the other hand, in the case of the production of an on-shell Higgs boson through a b-quark loop, $\Delta^{(2,0)} \approx 10 \%$. Furthermore, the difference grows rapidly with the Higgs-boson offshellness, $\sqrt{s}$. Hence, the approximation is rather poor for b-quark loops. In the same figure, we also show $\Delta^{(2,0)}$ using the improved Padé approximant of ref. [26]. We note that 

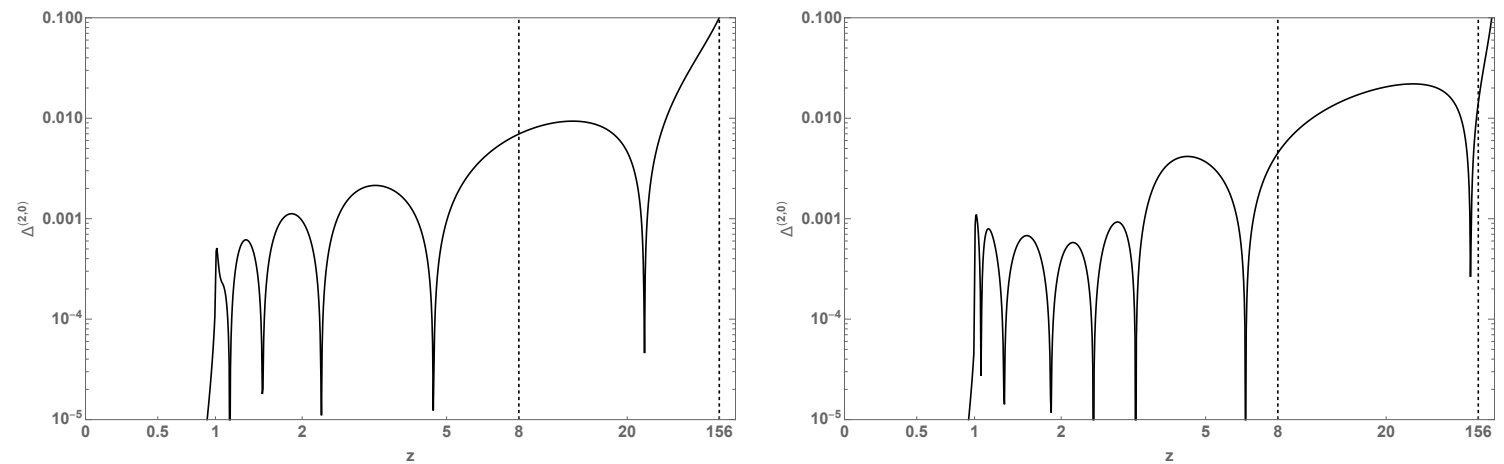

Figure 3. Relative difference, eq. (4.1), between the Padé approximation of the three-loop coefficient of the finite remainder $\mathcal{C}_{I}^{(2)}$ from refs. [9] (left panel) and [26] (right panel) and the exact result at $n_{l}=0, L_{\mu}=0 . z \approx 8$ corresponds to a $\sqrt{s}=1 \mathrm{TeV}$ Higgs boson produced through a top-quark loop, whereas $z \approx 156$ corresponds to an on-shell Higgs boson produced through a b-quark loop.
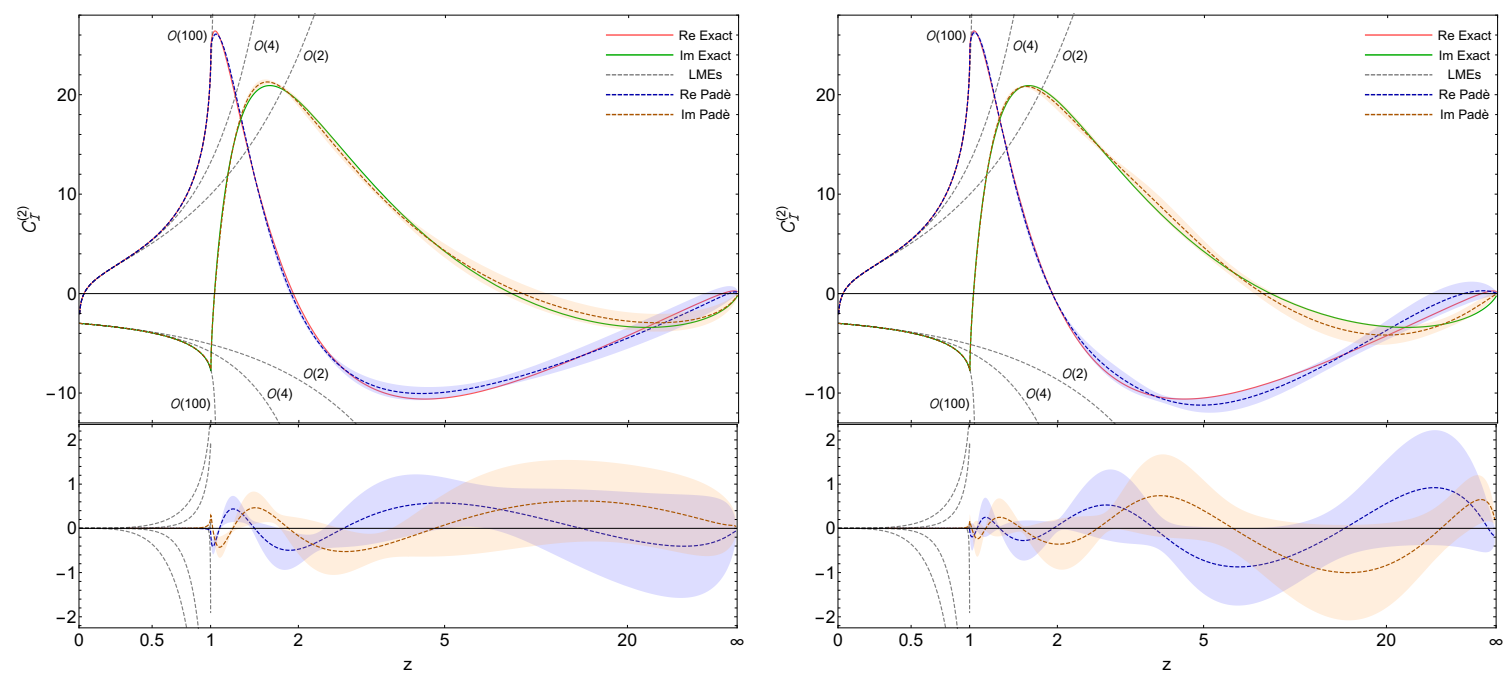

Figure 4. Comparison of the three-loop coefficient of the finite remainder, eq. (2.9), at $n_{l}=5$, $L_{\mu}=0$ (five massless quarks, renormalisation scale $\mu^{2}=-s$ ), with the default Padé approximation, [6,1], constructed in ref. [9] (left panel) and improved to [7, 1] in ref. [26] (right panel), as function of $z=s / 4 M^{2}$ with $\sqrt{s}$ the center-of-mass energy of the Higgs boson and $M$ the mass of the single massive quark. The bands correspond to the uncertainty of the Padé approximations as estimated in refs. [9] and [26]. The lower plot shows the absolute difference between the approximation and the exact result. Also shown is the large-mass expansion (LME) of the three-loop coefficient of the finite remainder truncated at $\mathcal{O}\left(z^{2}\right), \mathcal{O}\left(z^{4}\right)$ and $\mathcal{O}\left(z^{100}\right)$.

the approximation is now better for b-quarks. Nevertheless, $\Delta^{(2,0)}>10 \%$ for an off-shell Higgs boson of $400 \mathrm{GeV}$.

Our exact result is a sample of $\mathcal{C}_{I}^{(2)}$ values at nearly $200.000 z$ points. We have also determined the large-mass, threshold and high-energy expansions of $\mathcal{C}_{I}^{(2)}$ (see section 3). These three expansions cover most of the range of $z$ values within their convergence radii. In the supplemental material (see appendix D) to the present publication, we provide the large-mass expansion up to $\mathcal{O}\left(z^{100}\right)$ with exact coefficients, the threshold expansion up to 

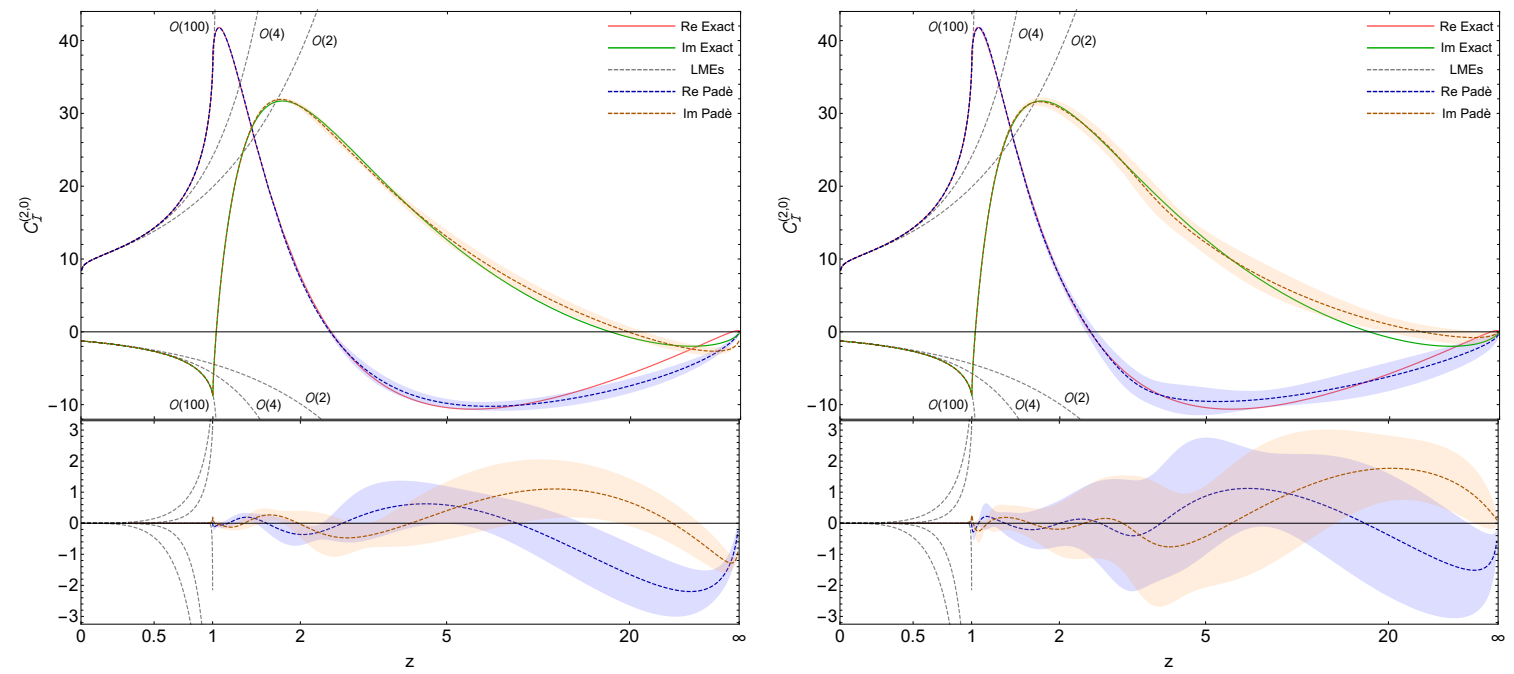

Figure 5. Same as figure 4 but with $n_{l}=0$.

$\mathcal{O}\left((1-z)^{20}\right)$ with numerical coefficients and the high-energy expansion up to $\mathcal{O}\left(1 / z^{8}\right)$ with numerical coefficients. The order at which the high-energy expansion has been truncated has been determined by the requirement that the numerical expansion coefficients have at least ten correct digits as determined in a conservative comparison of results obtained with two different starting points for the numerical solution of eqs. (3.3) and $y$ values eq. (3.5). The agreement of the truncated expansions with the exact result is demonstrated in figure 6 .

The domain of physical $z$ values may be compactified with the following mapping:

$$
z(\rho) \equiv \frac{4 \rho}{1-\rho}, \quad \rho(z)=\frac{z}{4+z}, \quad \rho \in(0,1) .
$$

The exact result for $\mathcal{C}_{I}^{(2,0)}$ is approximated to better than $10^{-5}$ relative to $\left|\mathcal{C}^{(2,0)}\right|$ as follows:

$$
\begin{aligned}
& 0<\rho<1 / 6 \quad \text { - large-mass expansion, appendix A and figure } 5 ; \\
& 1 / 6 \leq \rho<1 / 4 \quad-\quad \text { threshold expansion, appendix B and figure } 7 ; \\
& 1 / 4 \leq \rho<3 / 4 \quad-\quad \text { interpolation of a sample of numerical values, tables } 1 \text { and } 2 ; \\
& 3 / 4 \leq \rho<1 \quad \text { - high-energy expansion, appendix } \mathrm{C} \text { and figure } 8 .
\end{aligned}
$$

\section{Conclusions and outlook}

With the results presented in this work, the Higgs-gluon form factor is known exactly at three loops in QCD with a single massive quark. This is sufficient for applications to Higgs-boson hadroproduction in the five-flavour scheme, where the massive quark is the top. In this case, we have confirmed that an approach based on Padé approximants [9] is sufficient to obtain sub-percent precision for physical observables. On the other hand, our result removes any uncertainties on the value of the form factor present in ref. [9]. Once b-quark loops are considered at non-vanishing b-quark mass, our result becomes indispensable, since Padé approximants potentially induce errors on physical predictions in the ten-precent range. 


\begin{tabular}{|c|c|c|c|}
\hline$\rho$ & $\mathcal{C}_{I}^{(2,0)}$ & $\rho$ & $\mathcal{C}_{I}^{(2,0)}$ \\
\hline $1 / 4$ & $30.88057646+25.98752971 i$ & $3 / 8$ & $0.5489407632+28.08768382 i$ \\
$51 / 200$ & $29.16117325+27.19326399 i$ & $19 / 50$ & $-0.1268390632+27.6738637 i$ \\
$13 / 50$ & $27.46093382+28.21076656 i$ & $77 / 200$ & $-0.7713324763+27.25087704 i$ \\
$53 / 200$ & $25.78986495+29.06161664 i$ & $39 / 100$ & $-1.385714578+26.82008886 i$ \\
$27 / 100$ & $24.15526667+29.76456733 i$ & $79 / 200$ & $-1.971122667+26.38273798 i$ \\
$11 / 40$ & $22.56238753+30.33601069 i$ & $2 / 5$ & $-2.528655721+25.93994889 i$ \\
$7 / 25$ & $21.01490693+30.79034303 i$ & $81 / 200$ & $-3.05937427+25.49274254 i$ \\
$57 / 200$ & $19.51529601+31.14025791 i$ & $41 / 100$ & $-3.56430059+25.04204591 i$ \\
$29 / 100$ & $18.06509163+31.39698515 i$ & $83 / 200$ & $-4.044419136+24.58870072 i$ \\
$59 / 200$ & $16.6651066+31.5704884 i$ & $21 / 50$ & $-4.500677174+24.13347121 i$ \\
$3 / 10$ & $15.31559266+31.66963034 i$ & $17 / 40$ & $-4.933985559+23.67705121 i$ \\
$61 / 200$ & $14.01636758+31.70231201 i$ & $43 / 100$ & $-5.345219629+23.22007047 i$ \\
$31 / 100$ & $12.76691514+31.67559125 i$ & $87 / 200$ & $-5.735220182+22.76310042 i$ \\
$63 / 200$ & $11.566464+31.59578403 i$ & $11 / 25$ & $-6.104794506+22.30665937 i$ \\
$8 / 25$ & $10.4140502+31.46855168 i$ & $89 / 200$ & $-6.454717455+21.85121724 i$ \\
$13 / 40$ & $9.308566879+31.29897624 i$ & $9 / 20$ & $-6.785732545+21.39719977 i$ \\
$33 / 100$ & $8.248803784+31.0916259 i$ & $91 / 200$ & $-7.098553057+20.94499247 i$ \\
$67 / 200$ & $7.233478837+30.85061204 i$ & $23 / 50$ & $-7.393863147+20.49494408 i$ \\
$17 / 50$ & $6.261263221+30.57963911 i$ & $93 / 200$ & $-7.672318937+20.04736981 i$ \\
$69 / 200$ & $5.330801353+30.28204836 i$ & $47 / 100$ & $-7.934549597+19.60255421 i$ \\
$7 / 20$ & $4.44072674+29.96085629 i$ & $19 / 40$ & $-8.181158403+19.16075384 i$ \\
$71 / 200$ & $3.589674492+29.61878862 i$ & $12 / 25$ & $-8.412723764+18.72219971 i$ \\
$9 / 25$ & $2.776291163+29.2583102 i$ & $97 / 200$ & $-8.629800232+18.28709941 i$ \\
$73 / 200$ & $1.999242412+28.88165164 i$ & $49 / 100$ & $-8.832919461+17.85563919 i$ \\
$37 / 100$ & $1.257218899+28.49083281 i$ & $99 / 200$ & $-9.022591138+17.42798575 i$ \\
\hline
\end{tabular}

Table 1. Numerical values of the three-loop coefficient of the finite remainder $\mathcal{C}_{I}^{(2)}$ at $n_{l}=0$, $L_{\mu}=0$, for $1 / 4 \leq \rho \equiv z /(4+z)<1 / 2$.

For the presentation of our results, we have used two different infrared-renormalisation schemes. On the other hand, we have chosen to renormalise the Yukawa coupling in the on-shell scheme. Fortunately, a translation to any other scheme, e.g. $\overline{\mathrm{MS}}$, can be easily 


\begin{tabular}{|c|c|c|c|}
\hline$\rho$ & $\mathcal{C}_{I}^{(2,0)}$ & $\rho$ & $\mathcal{C}_{I}^{(2,0)}$ \\
\hline $1 / 2$ & $-9.199303854+17.00428794 i$ & $5 / 8$ & $-10.49655344+7.904442944 i$ \\
$101 / 200$ & $-9.363525841+16.58467833 i$ & $63 / 100$ & $-10.45672407+7.602825895 i$ \\
$51 / 100$ & $-9.515705879+16.16927502 i$ & $127 / 200$ & $-10.41175312+7.305965242 i$ \\
$103 / 200$ & $-9.656274528+15.75818283 i$ & $16 / 25$ & $-10.36179862+7.013842682 i$ \\
$13 / 25$ & $-9.785644947+15.35149431 i$ & $129 / 200$ & $-10.30701259+6.726439296 i$ \\
$21 / 40$ & $-9.904213631+14.94929071 i$ & $13 / 20$ & $-10.24754124+6.443735699 i$ \\
$53 / 100$ & $-10.01236111+14.55164295 i$ & $131 / 200$ & $-10.1835252+6.165712192 i$ \\
$107 / 200$ & $-10.11045262+14.15861246 i$ & $33 / 50$ & $-10.11509978+5.892348901 i$ \\
$27 / 50$ & $-10.19883876+13.77025201 i$ & $133 / 200$ & $-10.04239511+5.623625905 i$ \\
$109 / 200$ & $-10.2778561+13.38660649 i$ & $67 / 100$ & $-9.965536402+5.359523369 i$ \\
$11 / 20$ & $-10.34782779+13.00771356 i$ & $27 / 40$ & $-9.88464409+5.100021655 i$ \\
$111 / 200$ & $-10.40906411+12.63360429 i$ & $17 / 25$ & $-9.79983404+4.845101447 i$ \\
$14 / 25$ & $-10.46186303+12.2643038 i$ & $137 / 200$ & $-9.711217707+4.594743853 i$ \\
$113 / 200$ & $-10.5065107+11.89983176 i$ & $69 / 100$ & $-9.618902308+4.34893052 i$ \\
$57 / 100$ & $-10.54328201+11.54020288 i$ & $139 / 200$ & $-9.522990977+4.107643733 i$ \\
$23 / 40$ & $-10.57244099+11.18542744 i$ & $7 / 10$ & $-9.423582916+3.870866519 i$ \\
$29 / 50$ & $-10.59424131+10.83551164 i$ & $141 / 200$ & $-9.320773537+3.638582749 i$ \\
$117 / 200$ & $-10.60892672+10.49045807 i$ & $71 / 100$ & $-9.214654604+3.410777238 i$ \\
$59 / 100$ & $-10.61673142+10.15026603 i$ & $143 / 200$ & $-9.105314363+3.187435844 i$ \\
$119 / 200$ & $-10.61788051+9.814931857 i$ & $18 / 25$ & $-8.992837666+2.968545567 i$ \\
$3 / 5$ & $-10.6125903+9.484449303 i$ & $29 / 40$ & $-8.877306096+2.754094652 i$ \\
$121 / 200$ & $-10.60106877+9.158809768 i$ & $73 / 100$ & $-8.758798082+2.54407269 i$ \\
$61 / 100$ & $-10.58351579+8.838002595 i$ & $147 / 200$ & $-8.637389011+2.338470728 i$ \\
$123 / 200$ & $-10.56012358+8.52201532 i$ & $37 / 50$ & $-8.513151331+2.137281371 i$ \\
$31 / 50$ & $-10.5310769+8.210833902 i$ & $149 / 200$ & $-8.386154663+1.940498901 i$ \\
$5 / 8$ & $-10.49655344+7.904442944 i$ & $3 / 4$ & $-8.256465888+1.748119392 i$ \\
\hline
\end{tabular}

Table 2. Numerical values of the three-loop coefficient of the finite remainder $\mathcal{C}_{I}^{(2)}$ at $n_{l}=0$, $L_{\mu}=0$, for $1 / 2 \leq \rho \equiv z /(4+z) \leq 3 / 4$.

achieved thanks to the knowledge of one- and two-loop results in analytic form. This translation is independent of infrared renormalisation. 


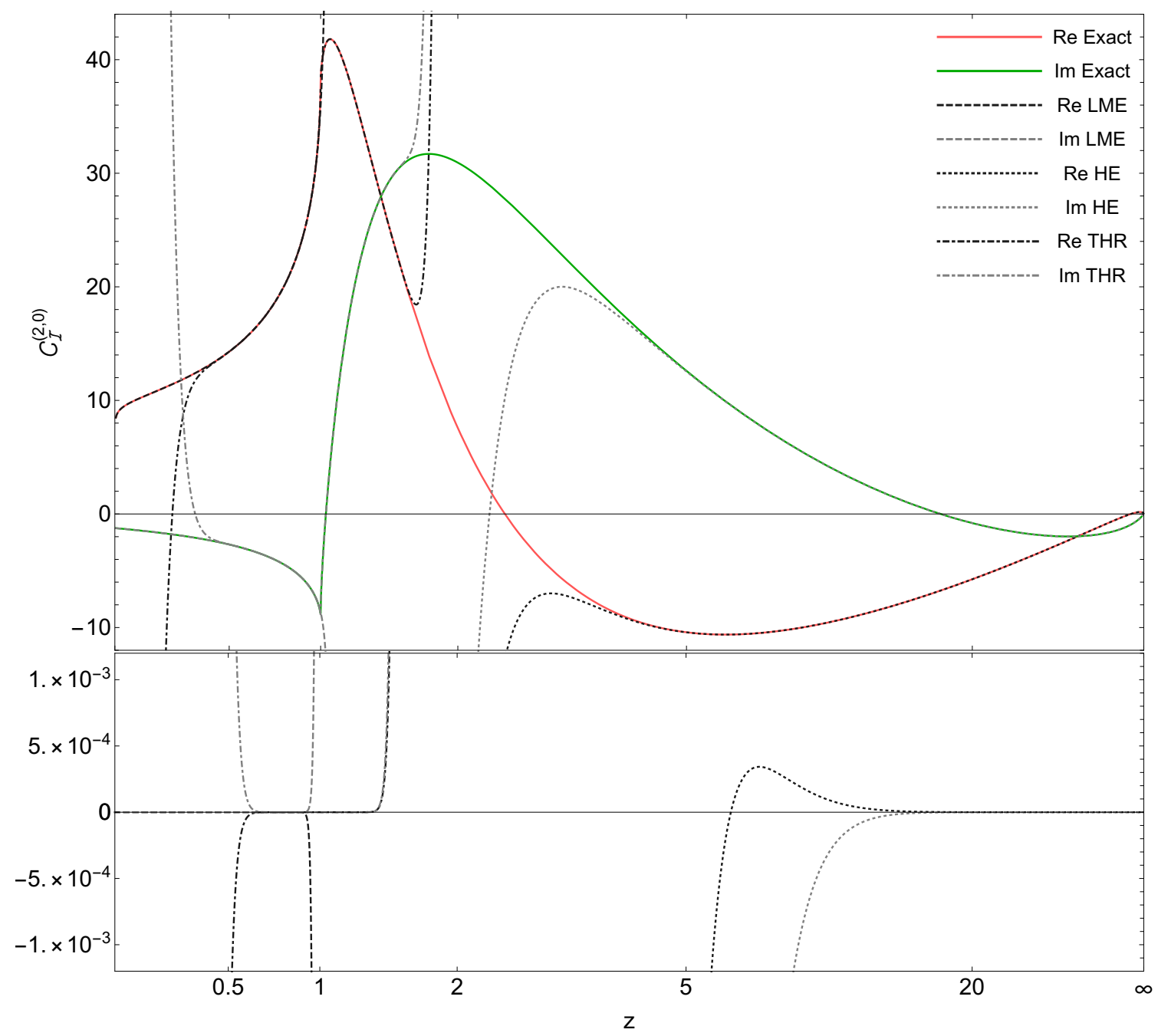

Figure 6. Comparison of the large-mass expansion (LME) truncated at $\mathcal{O}\left(z^{100}\right)$, threshold expansion (THR) truncated at $\mathcal{O}\left((1-z)^{20}\right)$ and high-energy expansion (HE) truncated at $\mathcal{O}\left(1 / z^{8}\right)$ with the exact result for the three-loop coefficient of the finite remainder $\mathcal{C}_{I}^{(2)}$ at $n_{l}=0, L_{\mu}=0$. The lower panel shows the absolute difference between the expansions and the exact result.

In principle, our calculation can also be used to obtain the form factor for the process $H \rightarrow \gamma \gamma$, as well as processes involving pseudo-scalars instead of a scalar. We intend to provide these results in forthcoming publications.

Finally, we stress that a complete knowledge of the form factor at three loops in the most general case requires the evaluation of diagrams with two different massive quarks. This can be achieved with numerical methods presented here, for example by fixing the ratio of the b- and top-quark masses. We leave this problem to future work.

Our results are available in computer readable form, see appendix D.

\section{Acknowledgments}

This work was supported by the Deutsche Forschungsgemeinschaft under grant 396021762 — TRR 257. 


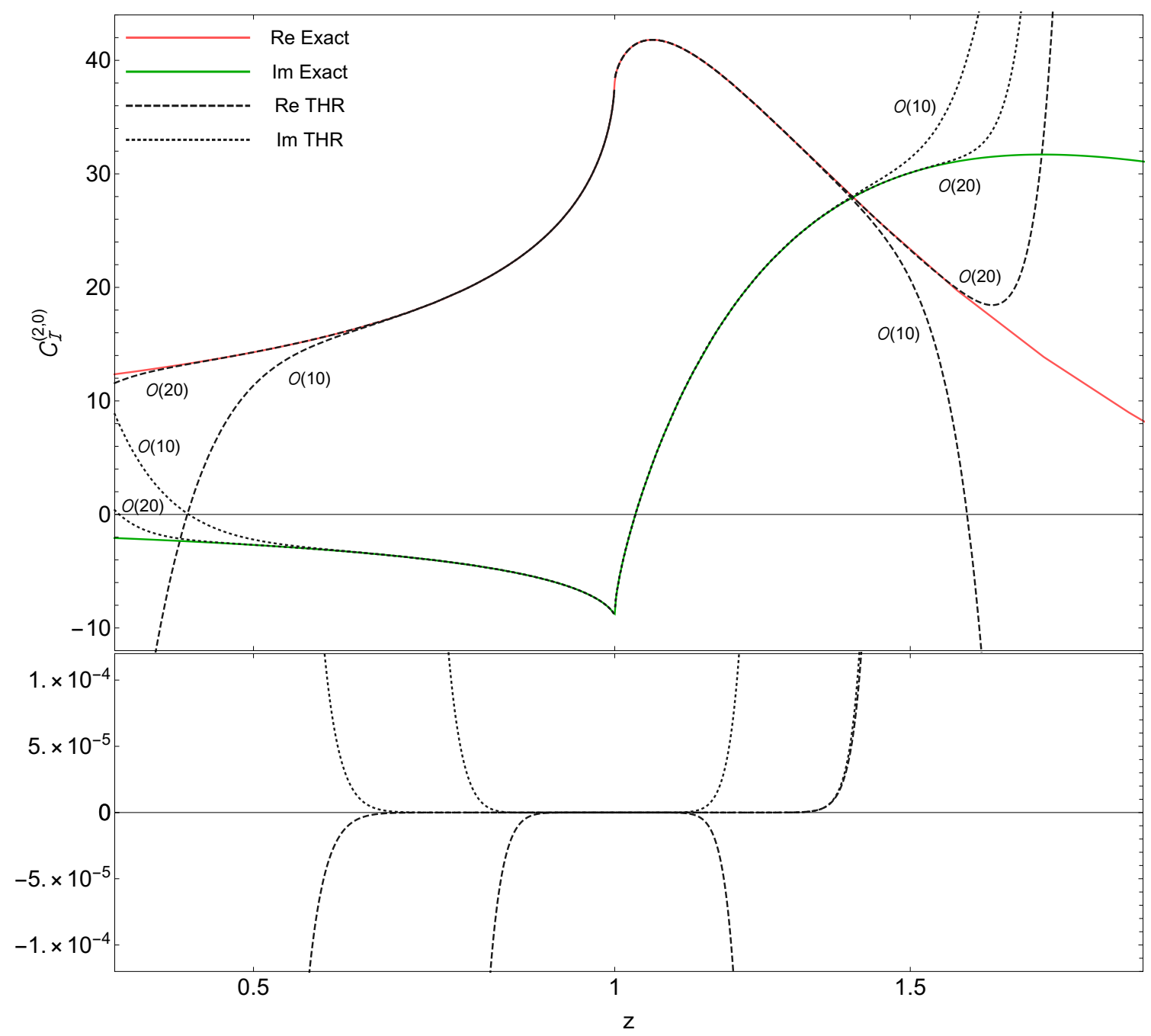

Figure 7. Comparison of the threshold expansion (THR) truncated at $\mathcal{O}\left((1-z)^{10}\right)$ and $\mathcal{O}\left((1-z)^{20}\right)$ with the exact result for the three-loop coefficient of the finite remainder $\mathcal{C}_{I}^{(2)}$ at $n_{l}=0, L_{\mu}=0$. The lower panel shows the absolute difference between the expansions and the exact result.

\section{A Large-mass expansion}

$$
\begin{aligned}
C_{I}^{(2,0)}= & \sum_{n=0}^{\infty}\left(a_{n, 0}+a_{n, 1} L_{s}\right) z^{n}, \quad L_{s} \equiv \ln \left(-\frac{s}{M^{2}}-i 0^{+}\right) \\
\mathcal{C}_{I}^{(2,0)}= & 10.1151523+0.3958333333 L_{s}+\left(4.778475062+0.6374228395 L_{s}\right) z \\
& +\left(3.071997564+0.3726469724 L_{s}\right) z^{2}+\left(2.113752253+0.2432786092 L_{s}\right) z^{3} \\
& +\left(1.549293473+0.1705037577 L_{s}\right) z^{4}+\left(1.188613713+0.1259718957 L_{s}\right) z^{5} \\
& +\left(0.9434907022+0.09730796586 L_{s}\right) z^{6}+(0.7698982981 \\
& \left.+0.07720332487 L_{s}\right) z^{7}+\left(0.6413834263+0.06309263508 L_{s}\right) z^{8}
\end{aligned}
$$




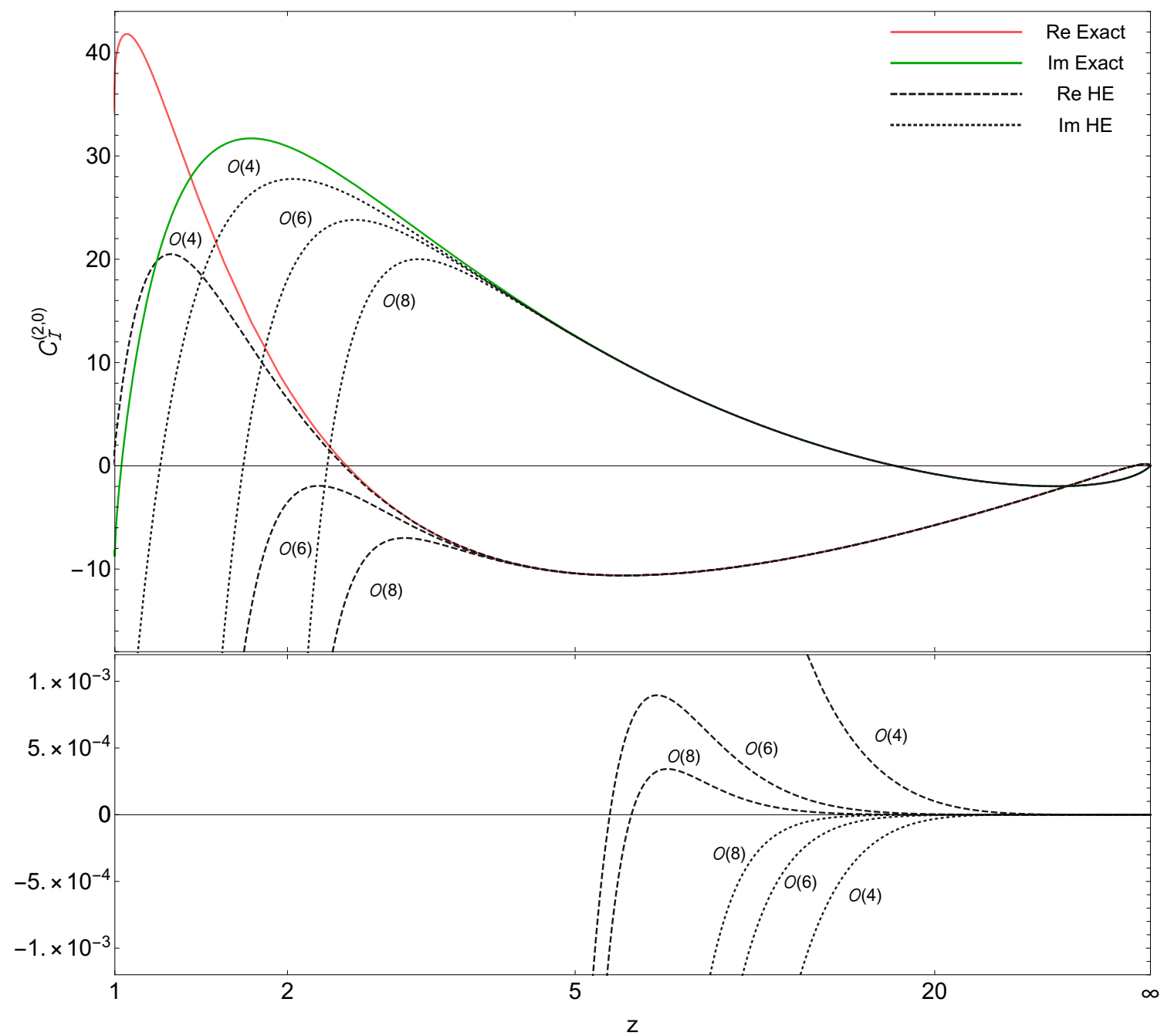

Figure 8. Comparison of the high-energy expansion (HE) truncated at $\mathcal{O}\left(1 / z^{4}\right), \mathcal{O}\left(1 / z^{6}\right)$ and $\mathcal{O}\left(1 / z^{8}\right)$ with the exact result for the three-loop coefficient of the finite remainder $\mathcal{C}_{I}^{(2)}$ at $n_{l}=0$, $L_{\mu}=0$. The lower panel shows the absolute difference between the expansions and the exact result.

$$
\begin{aligned}
& +\left(0.5441670543+0.05233299715 L_{s}\right) z^{9}+(0.4680660377 \\
& \left.+0.0443515761 L_{s}\right) z^{10}+\left(0.4078535762+0.0379145141 L_{s}\right) z^{11} \\
& +\left(0.3588441671+0.03295598554 L_{s}\right) z^{12}+(0.3187910863 \\
& \left.+0.02879246954 L_{s}\right) z^{13}+\left(0.2852395627+0.02549720532 L_{s}\right) z^{14} \\
& +\left(0.2571429415+0.02264475648 L_{s}\right) z^{15}+(0.2330827097 \\
& \left.+0.02034099839 L_{s}\right) z^{16}+\left(0.2125481506+0.01829860319 L_{s}\right) z^{17} \\
& +\left(0.1946546291+0.01662316981 L_{s}\right) z^{18}+(0.1791494756
\end{aligned}
$$




$$
\begin{aligned}
& \left.+0.01510884572 L_{s}\right) z^{19}+\left(0.165446505+0.01385124062 L_{s}\right) z^{20} \\
& +\left(0.1534242422+0.01269623978 L_{s}\right) z^{21}+(0.1426747173 \\
& \left.+0.01172751873 L_{s}\right) z^{22}+\left(0.1331457079+0.0108257358 L_{s}\right) z^{23} \\
& +\left(0.1245416017+0.01006326825 L_{s}\right) z^{24}+(0.1168475512 \\
& \left.+0.009345212464 L_{s}\right) z^{25}+\left(0.1098420694+0.008734026363 L_{s}\right) z^{26} \\
& +\left(0.1035305936+0.00815260668 L_{s}\right) z^{27}+(0.09774245353 \\
& \left.+0.007654956458 L_{s}\right) z^{28}+\left(0.09249392018+0.007177321845 L_{s}\right) z^{29} \\
& +\left(0.08765032243+0.006766580255 L_{s}\right) z^{30}+(0.08323342197 \\
& \left.+0.006369234271 L_{s}\right) z^{31}+\left(0.07913478115+0.006026173094 L_{s}\right) z^{32} \\
& +\left(0.07537859115+0.005691941208 L_{s}\right) z^{33}+(0.07187600638 \\
& \left.+0.005402388748 L_{s}\right) z^{34}+\left(0.068651888+0.005118475844 L_{s}\right) z^{35} \\
& +\left(0.06563233955+0.004871797509 L_{s}\right) z^{36}+(0.06284189053 \\
& \left.+0.004628509056 L_{s}\right) z^{37}+\left(0.06021826802+0.004416595976 L_{s}\right) z^{38} \\
& +\left(0.05778511593+0.004206475477 L_{s}\right) z^{39}+(0.0554893509 \\
& \left.+0.004023055687 L_{s}\right) z^{40}+\left(0.05335344447+0.003840290207 L_{s}\right) z^{41} \\
& +\left(0.05133168673+0.003680449807 L_{s}\right) z^{42}+(0.04944524976 \\
& \left.+0.003520452297 L_{s}\right) z^{43}+\left(0.04765441847+0.003380296411 L_{s}\right) z^{44} \\
& +\left(0.04597903331+0.003239407083 L_{s}\right) z^{45}+(0.04438431107 \\
& \left.+0.003115815786 L_{s}\right) z^{46}+\left(0.04288878386+0.002991085231 L_{s}\right) z^{47} \\
& +\left(0.04146176925+0.002881535172 L_{s}\right) z^{48}+(0.04012054547 \\
& \left.+0.00277056456 L_{s}\right) z^{49}+\left(0.03883787271+0.002672996802 L_{s}\right) z^{50} \\
& +\left(0.03762984606+0.002573818605 L_{s}\right) z^{51}+(0.03647214107 \\
& \left.+0.002486539456 L_{s}\right) z^{52}+\left(0.03537974752+0.002397527326 L_{s}\right) z^{53} \\
& +\left(0.03433082942+0.002319133078 L_{s}\right) z^{54}+(0.03333935067 \\
& \left.+0.002238933009 L_{s}\right) z^{55}+\left(0.03238561472+0.00216825219 L_{s}\right) z^{56} \\
& +\left(0.0314826373+0.002095729392 L_{s}\right) z^{57}+(0.03061257345
\end{aligned}
$$




$$
\begin{aligned}
& \left.+0.002031778036 L_{s}\right) z^{58}+\left(0.0297875648+0.001965975545 L_{s}\right) z^{59} \\
& +\left(0.02899137863+0.001907922209 L_{s}\right) z^{60}+(0.028235352 \\
& \left.+0.001848028357 L_{s}\right) z^{61}+\left(0.02750466385+0.001795166544 L_{s}\right) z^{62} \\
& +\left(0.02680991123+0.001740489184 L_{s}\right) z^{63}+(0.02613751555 \\
& \left.+0.001692215416 L_{s}\right) z^{64}+\left(0.02549739331+0.001642161338 L_{s}\right) z^{65} \\
& +\left(0.02487706468+0.001597957601 L_{s}\right) z^{66}+(0.02428582021 \\
& \left.+0.001552015981 L_{s}\right) z^{67}+\left(0.02371215609+0.00151143557 L_{s}\right) z^{68} \\
& +\left(0.02316478676+0.001469164564 L_{s}\right) z^{69}+(0.02263307899 \\
& \left.+0.001431820595 L_{s}\right) z^{70}+\left(0.02212521664+0.001392836423 L_{s}\right) z^{71} \\
& +\left(0.02163134597+0.001358392448 L_{s}\right) z^{72}+(0.02115916176 \\
& \left.+0.001322360441 L_{s}\right) z^{73}+\left(0.02069951073+0.001290522743 L_{s}\right) z^{74} \\
& +\left(0.02025963637+0.001257149966 L_{s}\right) z^{75}+(0.01983101697 \\
& \left.+0.001227661193 L_{s}\right) z^{76}+\left(0.01942047911+0.001196690333 L_{s}\right) z^{77} \\
& +\left(0.01902007236+0.001169324218 L_{s}\right) z^{78}+(0.01863623774 \\
& \left.+0.00114052849 L_{s}\right) z^{79}+\left(0.01826154308+0.00111508544 L_{s}\right) z^{80} \\
& +\left(0.01790207242+0.001088264332 L_{s}\right) z^{81}+(0.01755086504 \\
& \left.+0.001064567732 L_{s}\right) z^{82}+\left(0.01721367407+0.001039543422 L_{s}\right) z^{83} \\
& +\left(0.01688396883+0.001017436529 L_{s}\right) z^{84}+(0.01656719533 \\
& \left.+0.0009940508608 L_{s}\right) z^{85}+\left(0.01625721611+0.000973394174 L_{s}\right) z^{86} \\
& +\left(0.01595919178+0.000951506098 L_{s}\right) z^{87}+(0.01566734531 \\
& \left.+0.0009321751265 L_{s}\right) z^{88}+\left(0.0153865718+0.0009116585178 L_{s}\right) z^{89} \\
& +\left(0.01511142531+0.00089354188 L_{s}\right) z^{90}+(0.01484655363 \\
& \left.+0.0008742836775 L_{s}\right) z^{91}+\left(0.01458681559+0.0008572814757 L_{s}\right) z^{92} \\
& +\left(0.0143366284+0.0008391800862 L_{s}\right) z^{93}+(0.01409113199 \\
& \left.+0.0008232025116 L_{s}\right) z^{94}+\left(0.01385452828+0.0008061664372 L_{s}\right) z^{95} \\
& +\left(0.01362221703+0.0007911325696 L_{s}\right) z^{96}+(0.01339819894
\end{aligned}
$$




$$
\begin{aligned}
& \left.+0.0007750792236 L_{s}\right) z^{97}+\left(0.01317811431+0.000760915994 L_{s}\right) z^{98} \\
& +\left(0.01296577557+0.0007457706765 L_{s}\right) z^{99}+(0.01275704613 \\
& \left.+0.0007324119688 L_{s}\right) z^{100}+\mathcal{O}\left(z^{101}\right) .
\end{aligned}
$$

The exact expansion coefficients are provided in the supplementary material. We agree with refs. [6, 7] up to $\mathcal{O}\left(z^{4}\right)$ and with ref. [9] up to $\mathcal{O}\left(z^{6}\right)$.

\section{B Threshold expansion}

$$
\begin{aligned}
C_{I}^{(2,0)}= & \sum_{n=0}^{\infty}\left(b_{n, 0}+b_{n, 1} L_{t}+b_{n, 2} L_{t}^{2}\right) t^{n}, \\
L_{t} \equiv & \ln (1-z), \quad t \equiv \sqrt{1-z}=\exp \left(L_{t} / 2\right), \\
\mathcal{C}_{I}^{(2,0)}= & 38.29655119-8.9070147 i-29.55840851 t+(9.112936321-68.1395365 i \\
& \left.+(14.16269653-28.42242029 i) L_{t}-4.523568684 L_{t}^{2}\right) t^{2}+(-20.55378026 \\
& \left.+133.7985485 i-26.60436928 L_{t}\right) t^{3}+(-25.39554578-239.3964484 i \\
& \left.+(14.71881407-18.94828019 i) L_{t}-8.864366916 L_{t}^{2}\right) t^{4}+(22.88555562 \\
& +311.994478 i+(-43.65929113-30.41485955 i) L_{t}+(-0.3490658504 \\
& \left.+7.402203301 i) L_{t}^{2}\right) t^{5}+(-122.1397994-392.2909322 i+(6.009726459 \\
& \left.+13.26379614 i) L_{t}-5.516621472 L_{t}^{2}\right) t^{6}+(140.6543286+457.2900946 i \\
& \left.+(-70.34961079-68.49797789 i) L_{t}+(2.520477069+19.98594891 i) L_{t}^{2}\right) t^{7} \\
& +\left(-310.5867852-492.0494746 i+(-6.024184876+62.80001436 i) L_{t}\right. \\
& \left.+7.272523314 L_{t}^{2}\right) t^{8}+(359.8673214+541.6828656 i+(-116.1605627 \\
& \left.-105.7705087 i) L_{t}+(10.80021746+36.50872414 i) L_{t}^{2}\right) t^{9}+(-610.5588771 \\
& \left.-520.6092531 i+(-16.89694249+126.7730175 i) L_{t}+30.31073877 L_{t}^{2}\right) t^{10} \\
& +\left(700.264643+549.2184102 i+(-185.5662205-138.0922834 i) L_{t}\right. \\
& \left.+(26.001469+56.2053409 i) L_{t}^{2}\right) t^{11}+(-1036.677064-465.6193352 i \\
& \left.+(-22.72070802+203.3453965 i) L_{t}+64.09089853 L_{t}^{2}\right) t^{12}+(1177.227509 \\
& +468.516149 i+(-279.6079413-163.1047377 i) L_{t}+(49.23219084 \\
& \left.+78.56007164 i) L_{t}^{2}\right) t^{13}+(-1600.347449-317.3641993 i+(-19.90826165 \\
& \left.+291.2249078 i) L_{t}+108.9603809 L_{t}^{2}\right) t^{14}+(1803.642376+290.4177751 i \\
& \left.+(-396.3597986-179.328259 i) L_{t}+(81.34424135+103.2021919 i) L_{t}^{2}\right) t^{15} \\
& +3.96
\end{aligned}
$$


$+\left(-2310.834851-67.58556999 i+(-5.061769755+389.4423686 i) L_{t}\right.$ $\left.+165.1852917 L_{t}^{2}\right) t^{16}+(2591.104007+7.032756569 i+(-530.7745415$ - 185.7721012i) $\left.L_{t}+(123.0180749+129.8524061 i) L_{t}^{2}\right) t^{17}+(-3175.897771$ $\left.+291.0629642 i+(25.07572246+497.2376355 i) L_{t}+232.9804921 L_{t}^{2}\right) t^{18}$ $+\left(3550.803491-388.7191604 i+(-674.0712248-181.7412648 i) L_{t}\right.$ $\left.+(174.8140502+158.2927098 i) L_{t}^{2}\right) t^{19}+(-4202.187543+765.3035663 i$ $\left.+(73.64516718+613.9945923 i) L_{t}+312.5257534 L_{t}^{2}\right) t^{20}+(4694.305121$ $-903.3548866 i+(-812.6741315-166.7311215 i) L_{t}+(237.2049629$ $\left.+188.348174 i) L_{t}^{2}\right) t^{21}+(-5395.511981+1361.401213 i+(143.6864402$ $\left.+739.2011425 i) L_{t}+403.9753603 L_{t}^{2}\right) t^{22}+(6034.345387-1542.973831 i$ $\left.+(-926.6236752-140.3655593 i) L_{t}+(310.5975616+219.8752962 i) L_{t}^{2}\right) t^{23}$ $+\left(-6761.017023+2085.268299 i+(238.1485965+872.4231173 i) L_{t}\right.$ $\left.+507.4642605 L_{t}^{2}\right) t^{24}+(7585.760424-2313.350376 i+(-987.3075061$ $\left.-102.3585515 i) L_{t}+(395.3473322+252.7542072 i) L_{t}^{2}\right) t^{25}+(-8303.316472$ $\left.+2942.534197 i+(359.897675+1013.286463 i) L_{t}+623.1122305 L_{t}^{2}\right) t^{26}$ $+\left(9366.647246-3219.995987 i+(-954.2825083-52.48916829 i) L_{t}\right.$ $\left.+(491.7690072+286.8832593 i) L_{t}^{2}\right) t^{27}+(-10026.58756+3938.593854 i$ $\left.+(511.7230633+1161.464627 i) L_{t}+751.026828 L_{t}^{2}\right) t^{28}+(11399.88074$ $-4268.202962 i+(-770.8622683+9.415287382 i) L_{t}+(600.1442562$ $\left.+322.175152 i) L_{t}^{2}\right) t^{29}+(-11934.64323+5078.643159 i+(696.3429187$ $\left.+1316.669338 i) L_{t}+891.3055595 L_{t}^{2}\right) t^{30}+(13715.1406-5463.076486 i$ $\left.+(-358.0179892+83.49101847 i) L_{t}+(720.7274605+358.554085 i) L_{t}^{2}\right) t^{31}$ $+\left(-14030.98797+6367.70559 i+(916.4089125+1478.643714 i) L_{t}\right.$ $\left.+1044.037518 L_{t}^{2}\right) t^{32}+(16351.65146-6809.558924 i+(394.0297813$ $\left.+169.8461219 i) L_{t}+(853.750144+395.953618 i) L_{t}^{2}\right) t^{33}+(-16318.86196$ $\left.+7810.652869 i+(1174.510449+1647.156969 i) L_{t}+1209.304654 L_{t}^{2}\right) t^{34}$ $+\left(19361.90922-8312.448766 i+(1637.383024+268.5667181 i) L_{t}\right.$ $\left.+(999.4244394+434.3150324 i) L_{t}^{2}\right) t^{35}+(-18801.27633+9412.2214 i$ 


$$
\begin{aligned}
& \left.+(1473.178459+1822.0003 i) L_{t}+1387.182774 L_{t}^{2}\right) t^{36}+(22816.76328 \\
& -9976.415748 i+(3582.371933+379.721518 i) L_{t}+(1157.945845 \\
& \left.+473.5860544 i) L_{t}^{2}\right) t^{37}+(-21481.04193+11177.0256 i+(1814.888837 \\
& \left.+2002.983613 i) L_{t}+1577.742354 L_{t}^{2}\right) t^{38}+(26812.35732-11806.01319 i \\
& \left.+(6519.39523+503.3652836 i) L_{t}+(1329.495449+513.7198453 i) L_{t}^{2}\right) t^{39} \\
& +\left(-24360.79309+13109.56893 i+(2202.065568+2189.932902 i) L_{t}\right. \\
& \left.+1781.049193 L_{t}^{2}\right) t^{40}+\mathcal{O}\left(t^{41}\right) .
\end{aligned}
$$

We agree with ref. [10] for the coefficients of the first three non-analytic terms:

$$
b_{1,0}=-\frac{2 \pi^{3}}{27}\left(3+\pi^{2}\right), \quad b_{2,1}=\frac{\pi^{2}}{216}\left(458-15 \pi^{2}\right)+2 \pi i b_{2,2} \quad \text { and } \quad b_{2,2}=-\frac{99 \pi^{2}}{216} .
$$

We also provide a high precision result for the three-loop coefficient of the form-factor at threshold:

$$
\begin{aligned}
\mathcal{C}_{I}^{(2,0)}[z=1]=b_{0,0} \approx & +38.29655118857344308946576090253939 \\
& -8.907014700051001636660098822811295 i .
\end{aligned}
$$

\section{High-energy expansion}

$$
\begin{aligned}
C_{I}^{(2,0)}= & \sum_{n=1}^{\infty} \sum_{k=0}^{6} c_{n, k} L_{s}^{k} z^{-n}, \quad L_{s} \equiv \ln \left(-\frac{s}{M^{2}}-i 0^{+}\right) \\
\mathcal{C}_{I}^{(2,0)}= & \left(15.93205751-15.73631507 L_{s}-1.121722806 L_{s}^{2}+0.4035518803 L_{s}^{3}\right. \\
& \left.+0.08901988687 L_{s}^{4}-0.001736111111 L_{s}^{5}-0.0004822530864 L_{s}^{6}\right) z^{-1} \\
& +\left(0.06309685356+3.546786436 L_{s}-0.519984143 L_{s}^{2}-1.652739942 L_{s}^{3}\right. \\
& \left.-0.1240600623 L_{s}^{4}-0.004134114583 L_{s}^{5}+0.0005738811728 L_{s}^{6}\right) z^{-2} \\
& +\left(5.754168857+7.325854683 L_{s}-2.98120415 L_{s}^{2}+0.1651932919 L_{s}^{3}\right. \\
& \left.+0.003161112205 L_{s}^{4}-0.005756293403 L_{s}^{5}+0.000220630787 L_{s}^{6}\right) z^{-3} \\
& +\left(-10.66566232-10.56571524 L_{s}+10.33923567 L_{s}^{2}-0.313124275 L_{s}^{3}\right. \\
& \left.-0.1681889443 L_{s}^{4}+0.01392927758 L_{s}^{5}+0.0000316478588 L_{s}^{6}\right) z^{-4} \\
& +\left(-6.785278289+88.43750151 L_{s}-40.26616919 L_{s}^{2}+2.072111298 L_{s}^{3}\right. \\
& \left.+0.7341214981 L_{s}^{4}-0.04301260489 L_{s}^{5}-0.0003223560475 L_{s}^{6}\right) z^{-5} \\
& +\left(80.70142226-421.2250932 L_{s}+175.4294283 L_{s}^{2}-5.805171716 L_{s}^{3}\right.
\end{aligned}
$$




$$
\begin{aligned}
& \left.-3.062956746 L_{s}^{4}+0.1753725462 L_{s}^{5}+0.0009707792306 L_{s}^{6}\right) z^{-6} \\
& +\left(-486.1362845+2151.385984 L_{s}-853.4135303 L_{s}^{2}+26.4094276 L_{s}^{3}\right. \\
& \left.+14.84667539 L_{s}^{4}-0.8733492022 L_{s}^{5}-0.002646085951 L_{s}^{6}\right) z^{-7} \\
& +\left(2880.610148-11795.75065 L_{s}+4569.562554 L_{s}^{2}-140.0597361 L_{s}^{3}\right. \\
& \left.-78.99328343 L_{s}^{4}+4.758979333 L_{s}^{5}+0.008394276654 L_{s}^{6}\right) z^{-8}+\mathcal{O}\left(z^{-9}\right) .
\end{aligned}
$$

The value of the coefficient of the term proportional to $L_{s}^{6} / z$ agrees with refs. [27, 28], while the coefficient of the term proportional to $L_{s}^{5} / z$ has been confirmed in ref. [29].

\section{Supplemental material}

The supplementary material, conforming to Wolfram Mathematica format, provides the following results as second order polynomials in api $\equiv \alpha_{s} / \pi$ :

$\mathrm{CI}[\mathrm{z}, \mathrm{nl}, \mathrm{Lmu}]-\mathcal{C}_{I}$, eq. $(2.9)$;

$\mathrm{CZ}[z, \mathrm{nl}, \mathrm{Lmu}]-\mathcal{C}_{Z}$, eq. $(2.16)$

CItoCZ - conversion between infrared schemes, eq. (2.24).

The approximations used by the function $\mathrm{CI}[\mathrm{z}, \mathrm{nl}, \mathrm{Lmu}]$ are directly accessible with the following functions evaluated at $L_{\mu}=0$ :

C0 [z], C1I $[z], \quad$ C2I $[z, n l]-\mathcal{C}^{(0)}, \mathcal{C}_{I}^{(1)}$ and $\mathcal{C}_{I}^{(2)}$, eqs. (2.3) and (2.9);

C2ILMEn10 [z], C2ILMEnl1 [z] — large-mass expansion of $\mathcal{C}_{I}^{(2,0)}$ (appendix A) and $\mathcal{C}_{I}^{(2,1)}$;

C2ITHRn10[z], C2ITHRnl1 [z] - threshold expansion of $\mathcal{C}_{I}^{(2,0)}$ (appendix B) and $\mathcal{C}_{I}^{(2,1)}$;

C2IHEnlo[z], C2IHEnl1[z] — high-energy expansion of $\mathcal{C}_{I}^{(2,0)}$ (appendix C) and $\mathcal{C}_{I}^{(2,1)}$;

C2ITABnl0[z], C2ITABnl1 [z] - interpolation of $\mathcal{C}_{I}^{(2,0)}\left(\right.$ tables 1 and 2) and $\mathcal{C}_{I}^{(2,1)}$.

All functions require a numeric value for $z$. Finally, the large-mass expansion of $\mathcal{C}_{I}^{(2)}$ evaluated at $L_{\mu}=0$ with exact coefficients and dependence on $n_{l}$ is given by C2ILME.

The results correspond to QCD with $C_{A}=3, C_{F}=4 / 3, T_{F}=1 / 2$.

Note that we do not use the results of ref. [11] for $\mathcal{C}_{I}^{(2,1)}$ in the supplementary material.

Open Access. This article is distributed under the terms of the Creative Commons Attribution License (CC-BY 4.0), which permits any use, distribution and reproduction in any medium, provided the original author(s) and source are credited. 


\section{References}

[1] LHC Higgs Cross Section Working Group, Handbook of LHC Higgs Cross Sections: 4. Deciphering the Nature of the Higgs Sector, arXiv:1610.07922 [INSPIRE].

[2] M. Spira, A. Djouadi, D. Graudenz and P.M. Zerwas, Higgs boson production at the LHC, Nucl. Phys. B 453 (1995) 17 [hep-ph/9504378] [INSPIRE].

[3] R. Harlander and P. Kant, Higgs production and decay: Analytic results at next-to-leading order QCD, JHEP 12 (2005) 015 [hep-ph/0509189] [INSPIRE].

[4] C. Anastasiou, S. Beerli, S. Bucherer, A. Daleo and Z. Kunszt, Two-loop amplitudes and master integrals for the production of a Higgs boson via a massive quark and a scalar-quark loop, JHEP 01 (2007) 082 [hep-ph/0611236] [INSPIRE].

[5] U. Aglietti, R. Bonciani, G. Degrassi and A. Vicini, Analytic Results for Virtual QCD Corrections to Higgs Production and Decay, JHEP 01 (2007) 021 [hep-ph/0611266] [INSPIRE].

[6] R.V. Harlander and K.J. Ozeren, Top mass effects in Higgs production at next-to-next-to-leading order QCD: Virtual corrections, Phys. Lett. B 679 (2009) 467 [arXiv: 0907.2997$]$ [INSPIRE].

[7] A. Pak, M. Rogal and M. Steinhauser, Virtual three-loop corrections to Higgs boson production in gluon fusion for finite top quark mass, Phys. Lett. B 679 (2009) 473 [arXiv: 0907.2998] [INSPIRE].

[8] J. Davies, F. Herren and M. Steinhauser, Top Quark Mass Effects in Higgs Boson Production at Four-Loop Order: Virtual Corrections, Phys. Rev. Lett. 124 (2020) 112002 [arXiv: 1911.10214] [INSPIRE].

[9] J. Davies, R. Gröber, A. Maier, T. Rauh and M. Steinhauser, Top quark mass dependence of the Higgs boson-gluon form factor at three loops, Phys. Rev. D 100 (2019) 034017 [arXiv: 1906.00982] [INSPIRE].

[10] R. Gröber, A. Maier and T. Rauh, Reconstruction of top-quark mass effects in Higgs pair production and other gluon-fusion processes, JHEP 03 (2018) 020 [arXiv: 1709.07799] [INSPIRE].

[11] R.V. Harlander, M. Prausa and J. Usovitsch, The light-fermion contribution to the exact Higgs-gluon form factor in QCD, JHEP 10 (2019) 148 [arXiv:1907.06957] [INSPIRE].

[12] S. Catani, The Singular behavior of QCD amplitudes at two loop order, Phys. Lett. B 427 (1998) 161 [hep-ph/9802439] [INSPIRE].

[13] D. de Florian and J. Mazzitelli, A next-to-next-to-leading order calculation of soft-virtual cross sections, JHEP 12 (2012) 088 [arXiv:1209.0673] [INSPIRE].

[14] T. Becher and M. Neubert, Infrared singularities of scattering amplitudes in perturbative QCD, Phys. Rev. Lett. 102 (2009) 162001 [Erratum ibid. 111 (2013) 199905] [arXiv:0901.0722] [INSPIRE].

[15] C. Anastasiou, N. Deutschmann and A. Schweitzer, Quark mass effects in two-loop Higgs amplitudes, arXiv:2001.06295 [INSPIRE].

[16] K.G. Chetyrkin and F.V. Tkachov, Integration by Parts: The Algorithm to Calculate $\beta$-functions in 4 Loops, Nucl. Phys. B 192 (1981) 159 [InSPIRE].

[17] M. Czakon, DiaGen/IdSolver, unpublished. 
[18] S. Laporta, High precision calculation of multiloop Feynman integrals by difference equations, Int. J. Mod. Phys. A 15 (2000) 5087 [hep-ph/0102033] [InSPIRE].

[19] A.V. Kotikov, Differential equations method: New technique for massive Feynman diagrams calculation, Phys. Lett. B 254 (1991) 158 [INSPIRE].

[20] E. Remiddi, Differential equations for Feynman graph amplitudes, Nuovo Cim. A 110 (1997) 1435 [hep-th/9711188] [INSPIRE].

[21] M. Caffo, H. Czyz, S. Laporta and E. Remiddi, The Master differential equations for the two loop sunrise selfmass amplitudes, Nuovo Cim. A 111 (1998) 365 [hep-th/9805118] [INSPIRE].

[22] R. Boughezal, M. Czakon and T. Schutzmeier, NNLO fermionic corrections to the charm quark mass dependent matrix elements in $\bar{B} \rightarrow X_{s} \gamma$, JHEP 09 (2007) 072 [arXiv:0707.3090] [INSPIRE].

[23] https://www.boost.org.

[24] V.A. Smirnov, Applied asymptotic expansions in momenta and masses, Springer Tracts Mod. Phys. 177 (2002) 1 [INSPIRE].

[25] M. Czakon, P. Fiedler, T. Huber, M. Misiak, T. Schutzmeier and M. Steinhauser, The $\left(Q_{7}, Q_{1,2}\right)$ contribution to $\bar{B} \rightarrow X_{s} \gamma$ at $\mathcal{O}\left(\alpha_{\mathrm{s}}^{2}\right)$, JHEP 04 (2015) 168 [arXiv:1503.01791] [INSPIRE].

[26] J. Davies, R. Gröber, A. Maier, T. Rauh and M. Steinhauser, Padé approach to top-quark mass effects in gluon fusion amplitudes, arXiv: 1912.04097 [INSPIRE].

[27] T. Liu and A.A. Penin, High-Energy Limit of QCD beyond the Sudakov Approximation, Phys. Rev. Lett. 119 (2017) 262001 [arXiv:1709.01092] [InSPIRE].

[28] T. Liu and A.A. Penin, High-Energy Limit of Mass-Suppressed Amplitudes in Gauge Theories, JHEP 11 (2018) 158 [arXiv:1809.04950] [INSPIRE].

[29] C. Anastasiou and A.A. Penin, Light Quark Mediated Higgs Boson Threshold Production in the Next-to-Leading Logarithmic Approximation, arXiv:2004.03602 [INSPIRE]. 